\title{
Evaluation of the Thickness in Nanolayers Using the Transfer Matrix Method for Modeling the Spectral Reflectivity
}

\author{
Juan E. González-Ramírez, ${ }^{1}$ Juan Fuentes, ${ }^{2}$ Luis C. Hernández, ${ }^{3}$ and Luís Hernández² \\ ${ }^{1}$ Department of Physics and Mathematics, Autonomous University of Aguascalientes, Avenue Universidad \# 940, \\ Ciudad Universitaria, C. P. 20100 Aguascalientes, AGS, Mexico \\ ${ }^{2}$ Department of General Physics, Faculty of Physics, University of Havana, 10400 La Habana, Cuba \\ ${ }^{3}$ Institute of Sciences and Technology of the Materials, University of Havana, 10400 La Habana, Cuba \\ Correspondence should be addressed to Juan E. González-Ramírez, jegonza@correo.uaa.mx
}

Received 3 December 2008; Accepted 13 February 2009

Recommended by Lian Gao

\begin{abstract}
The reflectivity spectra have been traditionally used to determine the thicknesses in semiconductor films. However, thicknesses of nanofilms are not easy to evaluate because the interference fringes are not visible in the transparent region. In this paper, we present a computed method based on the transfer matrix (TM) which is used to match the calculated and experimental room temperature reflectivity spectra of the $\mathrm{ZnTe} / \mathrm{GaAs}$ films and to determine its thickness film values afterwards. The TM method needs only to know refraction indices and absorption coefficients as a function of wavelength for the film and the substrate. The thickness nanofilms evaluated by our method are in agreement with the values measured by ellipsometry, Rutherford backscattering spectroscopy and transmission electron microscopy techniques. The present procedure extends the application of the standard spectral reflectance technique to determine semiconductor nanolayer thicknesses.
\end{abstract}

Copyright (C) 2009 Juan E. González-Ramírez et al. This is an open access article distributed under the Creative Commons Attribution License, which permits unrestricted use, distribution, and reproduction in any medium, provided the original work is properly cited.

\section{Introduction}

Semiconductor nanolayers are profusely used in the modern technology, therefore to determine their thicknesses becomes an essential theme. The spectral reflectance has been extensively applied to measure the semiconductor layer thicknesses as it is carried out quickly: it is a nondestructive technique, and a special surface preparation of the sample is not necessary. These measurements are usually taken in the photon energy region, where the semiconductor layers are transparent, and distinct interference fringes are clearly observed. However, in the very thin semiconductor films, the spectral reflectance technique is rejected because the interference fringes do not become visible; there is no evidence of maxima or minima intensity: only broad modulations are observed.

The transfer matrix (TM) method has been widely used to determine the values of the optical constants and thickness of thin films from their reflectance spectra in the visible wavelength range [1-5]. The photon transport through the multilayered structure is usually described using the TM method. In this approximation, it is assumed that each individual layer can be characterized by a well-defined thickness and refractive index which depends on wavelengths. The general features of the reflection and transmission coefficients are theoretically reproduced by this method.

The TM method is critically tested in this work to obtain the computed reflection spectra which are matched with experimental data of $\mathrm{ZnTe} / \mathrm{GaAs}$ nanolayers in order to determine their thicknesses. Using the minimum squares method, the thickness value uncertainty of each layer is found out. To verify our results, a commercial program is used to compare with the TM reflection spectra showing a good agreement.

Likewise, we compare the $\mathrm{ZnTe} / \mathrm{GaAs}$ film thicknesses obtained by reflectivity using the TM method with the thicknesses reported for the same films measured by ellipsometry, transmission electron microscopy (TEM), and Rutherford backscattering spectroscopy (RBS) techniques. The present procedure is very useful because it extends the application of the standard spectral reflectance technique to determine semiconductor nanolayer thicknesses. 


\section{Model Details}

The TM method is shortly presented below. Stationary regimes of electromagnetic monochromatic plane waves impinge on a homogeneous and isotropic thin dielectric film between two semi-infinite transparent media. Taking into account the boundary conditions for electric $(E)$ and magnetic $(B)$ fields, a matrix equation is obtained which relates the fields at two adjacent boundaries:

$$
\left[\begin{array}{l}
E_{a} \\
B_{a}
\end{array}\right]=\left[\begin{array}{cc}
\cos \delta & i \operatorname{sen} \delta / \gamma_{1} \\
i \gamma_{1} \operatorname{sen} \delta & \cos \delta
\end{array}\right]\left[\begin{array}{l}
E_{b} \\
B_{b}
\end{array}\right],
$$

where $a$ and $b$ indices denote the two layer boundaries, $\delta=2 \pi n_{1} t \cos \chi / \lambda_{0}$ is the electromagnetic wave phase change arising from path length in the layer, $n_{1}$ is the layer refraction index, $t$ is the layer thickness, and $\chi$ is the refraction angle. The $\gamma_{1}$ coefficient takes two values:

$$
\begin{aligned}
& \gamma_{1}=n_{1} \sqrt{\varepsilon_{0} \mu_{0}} / \cos \chi, \\
& \gamma_{1}=n_{1} \sqrt{\varepsilon_{0} \mu_{0}} \cos \chi,
\end{aligned}
$$

when electric field is parallel or perpendicular to the plane of incidence, respectively. The $\varepsilon_{0}$ and $\mu_{0}$ constants have the usual physical meaning. The second-order matrix in (1) is the TM, and it is commonly denoted as

$$
M=\left[\begin{array}{ll}
m_{11} & m_{12} \\
m_{21} & m_{22}
\end{array}\right] .
$$

Using the above scheme, the amplitude coefficients of reflection $(\rho)$ and transmission $(\tau)$ are derived as a function of the matrix components:

$$
\begin{aligned}
& \rho=\frac{\gamma_{0} m_{11}+\gamma_{0} \gamma_{s} m_{12}-m_{21}-\gamma_{s} m_{22}}{\gamma_{0} m_{11}+\gamma_{0} \gamma_{s} m_{12}+m_{21}+\gamma_{s} m_{22}}, \\
& \tau=\frac{2 \gamma_{0}}{\gamma_{0} m_{11}+\gamma_{0} \gamma_{s} m_{12}+m_{21}+\gamma_{s} m_{22}},
\end{aligned}
$$

where the $\gamma_{0}$ and $\gamma_{s}$ coefficients are identical to the reported ones by Hecht [6], and they characterize the optical properties in the film and in the substrate, respectively. To find either $(\rho)$ or $(\tau)$ for any configuration of layers, it is only needed to compute the transfer matrices for each layer, multiply them, and then substitute the resulting matrix components into the above equations. Multiplying $(\rho)$ by its complex conjugate leads to the reflectance coefficient $R$ :

$$
R=\rho \times \rho^{*} .
$$

The present procedure consists in matching the computed reflectance coefficient obtained by (6) with experimental data. In order to determine the film thickness, the $t$ parameter is varied in (5), and the value that better matches computed and experimental reflectance coefficients is chosen. This better value of $t$ parameter is found by minimum squares method:

$$
\operatorname{dif}=R_{\exp }\left(\lambda_{i}\right)-R_{\text {com }}\left(\lambda_{i}\right),
$$

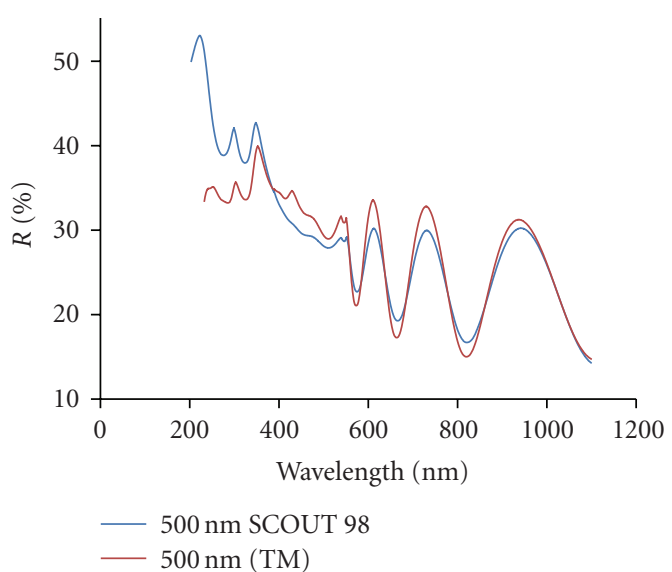

(a)

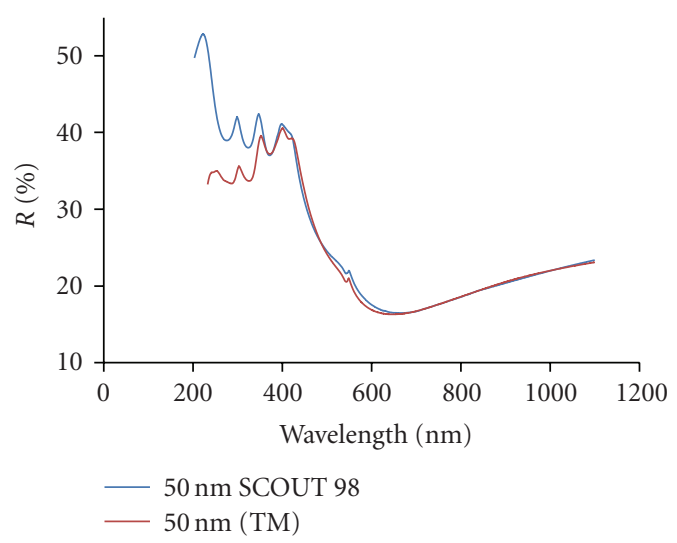

(b)

Figure 1: The ZnTe/GaAs reflectance spectra calculated by the TM method and by "SCOUT 98" commercial program, for two different thicknesses: (a) thin film, $t=500 \mathrm{~nm}$, and (b) very thin film, $t=$ $50 \mathrm{~nm}$.

where $R_{\exp }$ and $R_{\text {com }}$ are the experimental and computed reflectance coefficients, respectively, and the difference dif is taken at the same wavelength $\lambda_{i}$. The layer thickness that does the minimum sum of the quadratic difference for each wavelength is chosen. The uncertainty $\sigma$ in the thickness value determination was evaluated by the wellknown equation

$$
\sigma=\sqrt{\frac{\sum_{i=1}^{n}\left(R_{\exp _{i}}\left(\lambda_{i}\right)-R_{\mathrm{com}_{i}}\left(\lambda_{i}\right)\right)^{2}}{(n-2)}} .
$$

\section{Results and Discussions}

The reflectance spectra were recorded on $\mathrm{ZnTe} / \mathrm{GaAs}$ samples which were grown onto n-type GaAs (100) substrates in an isothermal close space configuration using elemental $\mathrm{Zn}$ and Te elemental sources [7]. Zinc telluride is a promising semiconductor material of II-VI groups for fabrication of high efficiency thin film solar cells and other optoelectronic devices due to its suitable intrinsic energy gap, $2.26 \mathrm{eV}$ [8]. The reflectance measurements in the visible range 
TABLE 1: Thickness evaluation using ellipsometry, RBS, TEM, and reflectivity spectra (present work) techniques.

\begin{tabular}{lr}
\hline & Thickness measurement $(\mathrm{nm})$ \\
\hline$(22 \pm 4)^{*}$ & $23^{* *}$ \\
$(59 \pm 5)^{*}$ & $60^{\dagger}$ \\
$(104 \pm 6)^{*}$ & $125^{\ddagger}$ \\
\hline
\end{tabular}

*Reflectivity; ${ }^{* *}$ Ellipsometry; ${ }^{\dagger} \mathrm{TEM} ;{ }^{\ddagger} \mathrm{RBS}$.

were measured by employing an UV-Vis unicam photospectrometer. The wavelength accuracy is $0.4 \mathrm{~nm}$, and the accuracy of the photometric measurements is $0.3 \%$. A tungsten halogen lamp was employed as light source in the visible range. This simple equipment allows, as it would be exposed, a great accuracy in the determination of the optical parameters of the samples measured.

The goal of the work is to determine the thickness in very thin films, where the light is not completely absorbed. For this reason, it is necessary to include in the reflectance spectra calculations, the ZnTe and GaAs refraction indices and the absorption coefficients as a function of wavelength, which its values were taken from $[9,10]$.

Figure 1 shows the $\mathrm{ZnTe} / \mathrm{GaAs}$ reflectance spectra calculated by the TM method, for two different thicknesses: (a) thin film, $t=500 \mathrm{~nm}$, where several interference fringes are observed, and (b) nanofilm, $t=50 \mathrm{~nm}$, where there are no interference fringes in the transparent photon energy region, only broad intensity modulation. Figure 1 also displays the reflectance spectra computed by a commercial program [11], showing a good agreement with TM spectra in the maximum, minimum, and critical point positions.

The experimental and computed reflectance spectra are shown in Figure 2 for the best fit: (a) $t=(22 \pm 4) \mathrm{nm}$ and (b) $t=(59 \pm 5) \mathrm{nm}$. The uncertainty values were calculated by the minimum squares method. Note the good agreement between both spectra, in particular the coincidence in the wavelength values for the critical points.

To verify our results, $\mathrm{ZnTe} / \mathrm{GaAs}$ film thickness values are compared with those measured by other techniques for the same layers [12]. Table 1 displays the good concordance between the film thickness values determined by reflectivity, using the TM method, and the values measured by ellipsometry, TEM, and RBS techniques. Similar results in the thickness film values were reported for very thin films [13]. However, these authors used a method based on the model dielectric function to fit the room temperature reflectivity spectra. This model makes use of numerous optical constants becoming troublesome, avoiding the evaluation of the uncertainty in the thickness determination.

The TM method using usual optical constants, refraction index, and absorption coefficient gives a simple procedure to determine, by the reflectivity spectra, the thicknesses of very thin films when the interference fringes in the transparent region are not observable.

\section{Conclusions}

A simple method to determine the thicknesses of very thin films by reflectivity spectra was described here. A procedure

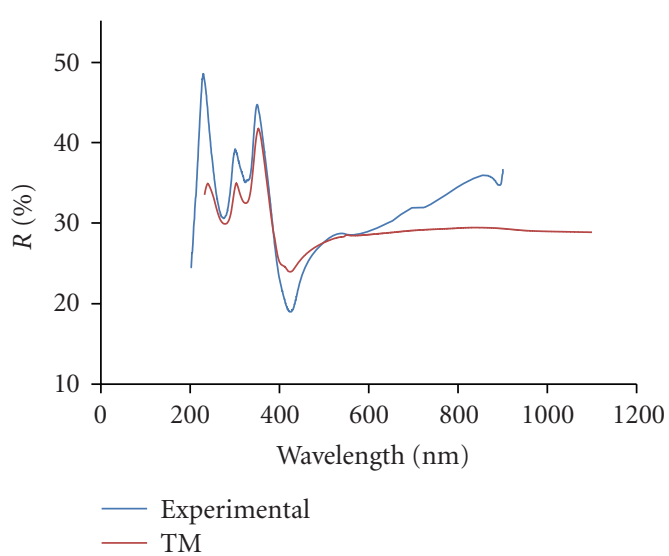

(a) $t=(22 \pm 4) \mathrm{nm}$

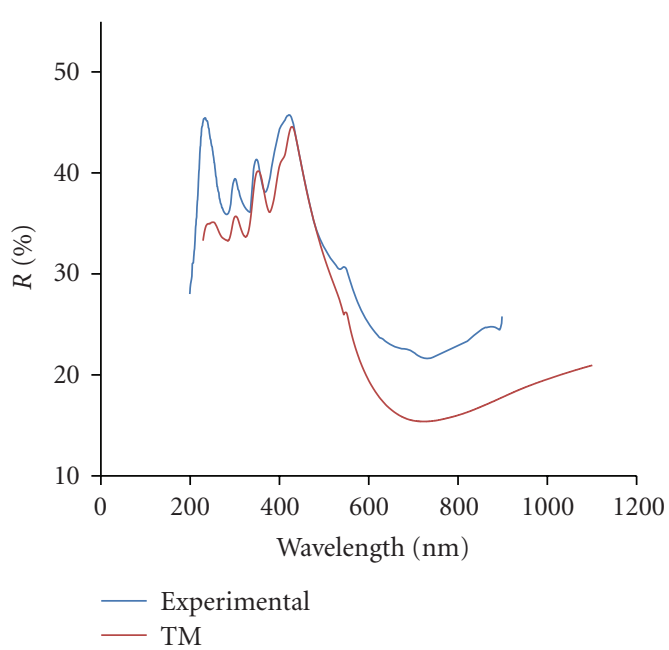

(b) $t=(59 \pm 5) \mathrm{nm}$

Figure 2: The experimental and computed reflectance spectra for the best fit: (a) $t=(22 \pm 4) \mathrm{nm}$, and (b) $t=(59 \pm 5) \mathrm{nm}$. The uncertainty values were calculated by the minimum squares method.

based on the TM is used to match the computed and experimental room temperature reflectivity spectra of the $\mathrm{ZnTe} / \mathrm{GaAs}$ films and to determine its thickness film values afterwards. The TM method needs only to know usual optical constants, that is, refraction indices and absorption coefficients as a function of wavelength for the film and the substrate. The thickness films evaluated by our method are in agreement with the values measured by ellipsometry, RBS, and TEM techniques. The present procedure extends the application of the standard spectral reflectance technique to determine semiconductor nanolayer thicknesses.

\section{References}

[1] A. K. S. Aqili and A. Maqsood, "Determination of thickness, refractive index, and thickness irregularity for semi-conductor thin films from transmission spectra," Applied Optics, vol. 41, no. 1, pp. 218-224, 2002.

[2] R. M. Bueno, J. F. Trigo, J. M. Martínez-Duart, E. Elizalde, and J. M. Sanz, "Study of the optical constants determination of 
thin films: dependence on theoretical assumptions," Journal of Vacuum Science and Technology A, vol. 13, no. 5, pp. 23782383, 1995.

[3] R. J. Martín-Palma, J. M. Martínez-Duart, and A. Macleod, "Determination of the optical constants of a semiconductor thin film employing the matrix method," IEEE Transactions on Education, vol. 43, no. 1, pp. 63-68, 2000.

[4] P. Pirasteh, Y. G. Boucher, J. Charrier, and Y. Dumeige, "A new approach based on transfer matrix formalism to characterize porous silicon layers by reflectometry," Physica Status Solidi (C), vol. 4, no. 6, pp. 1971-1975, 2007.

[5] J. M. Leger, S. A. Carter, B. Ruhstaller, et al., "Thicknessdependent changes in the optical properties of PPV- and PFbased polymer light emitting diodes," Physical Review B, vol. 68, no. 5, Article ID 054209, 6 pages, 2003.

[6] E. Hecht, Optics, Addison Wesley, New York, NY, USA, 1998.

[7] E. M. Larramendi, E. Purón, L. C. Hernández, et al., "Atomic layer epitaxy of ZnTe by isothermal closed space sublimation," Journal of Crystal Growth, vol. 223, no. 4, pp. 447-449, 2001.

[8] R. Sarma, N. Mazumdar, and H. L. Das, "Some spectral response characteristics of ZnTe thin films," Bulletin of Materials Science, vol. 29, no. 1, pp. 15-16, 2006.

[9] K. Sato and S. Adachi, "Optical properties of ZnTe," Journal of Applied Physics, vol. 73, no. 2, pp. 926-931, 1993.

[10] D. E. Aspnes and A. A. Studna, "Dielectric functions and optical parameters of $\mathrm{Si}, \mathrm{Ge}, \mathrm{GaP}, \mathrm{GaAs}, \mathrm{GaSb}$, InP, InAs, and InSb from 1.5 to $6.0 \mathrm{eV}$," Physical Review B, vol. 27, no. 2, pp. 985-1009, 1983.

[11] M. Theiss, "Hard- and software for optical spectroscopy "SCOUT 98"', Dr Bernhard-Klein-Str. 110, 52078 Aachen, Germany, www.mtheiss.com.

[12] L. C. Hernández, Growth of ZnTe semiconductor thin films onto GaAs and Si substrates by isotherm closed space sublimation. First intention of doping, M.S. thesis, University of Havana, Havana, Cuba, 2003.

[13] E. M. Larramendi, E. Purón, and O. de Melo, "Thickness measurement and optical properties of very thin $\mathrm{Zn}_{x} \mathrm{Cd}_{(1-x)} \mathrm{Te}$ layers," Semiconductor Science and Technology, vol. 17, no. 1, pp. 8-12, 2002. 

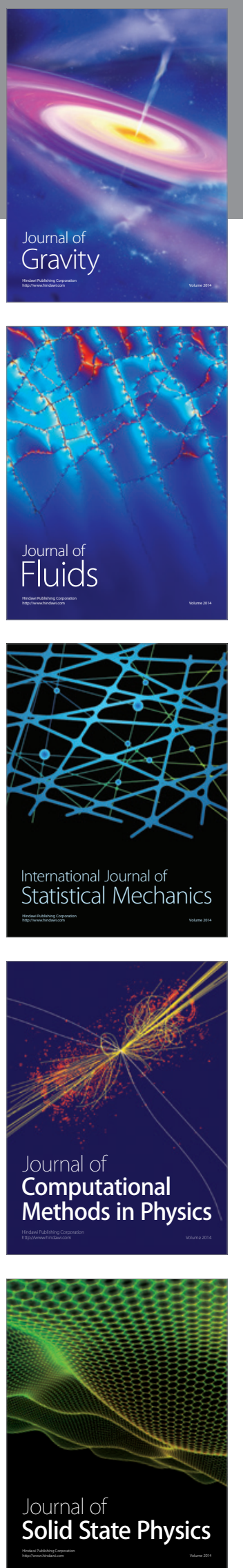

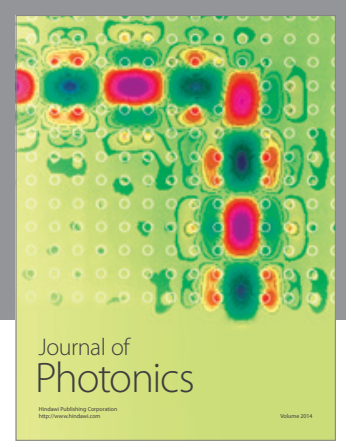

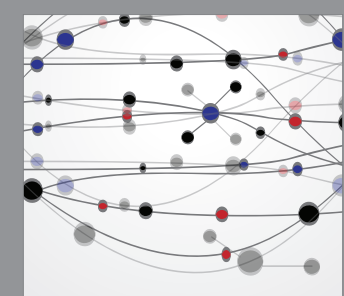

The Scientific World Journal
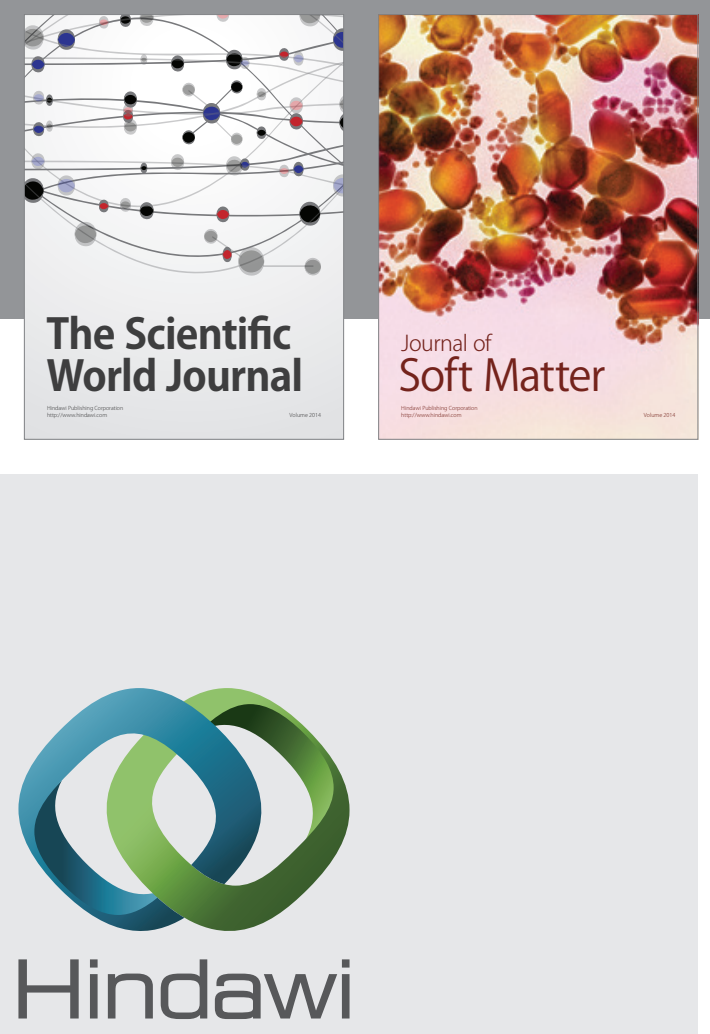

Submit your manuscripts at

http://www.hindawi.com
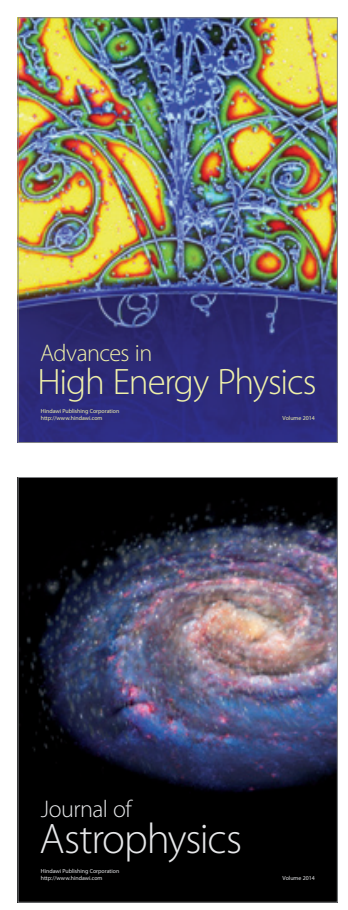
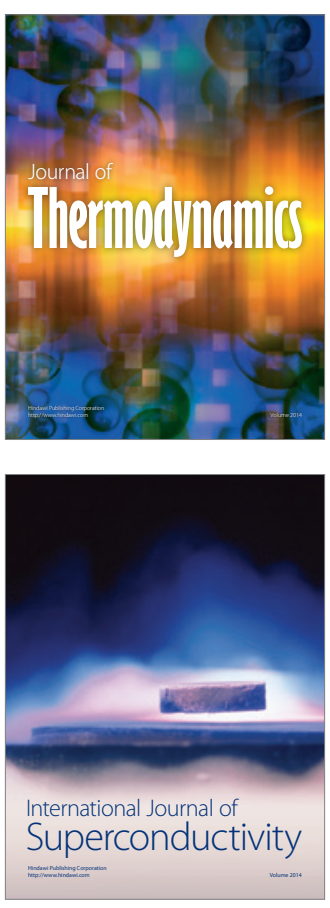
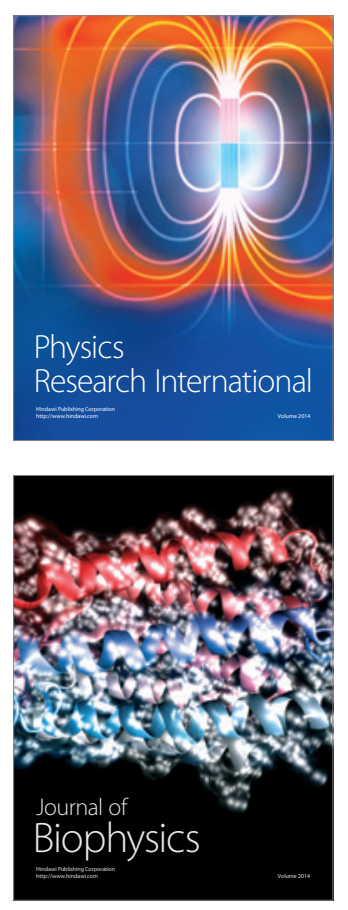
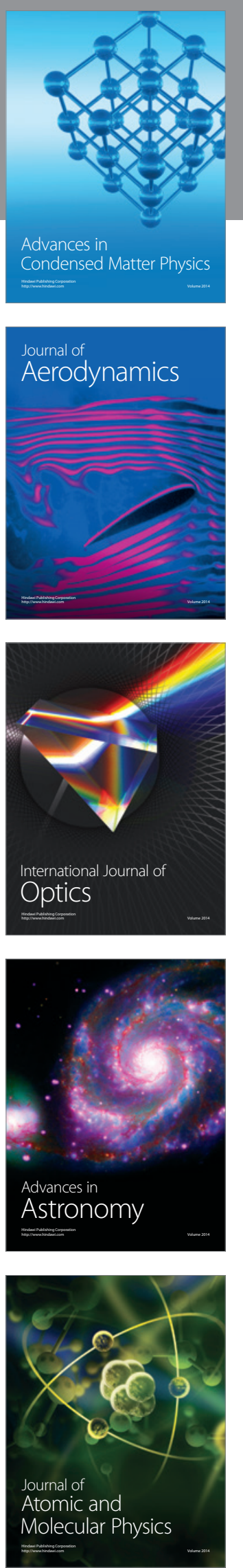